Le Lait (1985), 65 (1), 35-44

\title{
Etude de l'activité protéolytique d'une préparation microbienne destinée à accélérer l'affinage des fromages
}

\author{
par \\ Halina ZELAZOWSKA, L. JEDRYCHOWSKI et A. REPS
}

\section{Rés u m é}

On a étudié le processus de dégradation des protéines dans la pâte des fromages Edam, Tilsit et Kortowski par la préparation IPM, destinée à accélérer l'affinage des fromages.

L'activité de cette préparation a été comparée à l'activité protéolytique des enzymes coagulants. On a constaté que l'activité de la préparation IPM, à la concentration de $0,02 \% \mathrm{v} / \mathrm{v}$, était plus élevée que celle de la présure s'il s'agit de la libération des composés de poids moléculaire bas, tout en étant plus faible que celle de la Fromase, ces préparations étant utilisées à la concentration en usage pour la fabrication de fromages. L'intensité des modifications des protéines dépendait de la variété de fromage : elle était plus élevée pour les protéines des fromages Edam et Kortowski que pour celles du fromage Tilsit.

Mots clés

Pâte de fromage - Activité protéolytique - Accélération de l'affinage des fromages - Présure - Fromase.

\section{S u m m a r y}

STUDIES ON PROTEOLYTIC ACTIVITY OF MICROBIAL PREPARATION FOR CHEESE RIPENING ACCELERATION

Protein degradation in Tilsit, Edam and Kortowski cheese slurries by microbial preparation IPM for cheese ripening accele-

Institut du Génie et de la Biotechnologie Alimentaire de l'Université Agrotechnique d'Olsztyn (Pologne). 
ration was studied. The proteolytic activity of the preparation IPM was compared to the proteolytic activities of coagulating enzymes rennet and Fromase.

The activity of the preparation IPM at its concentration 0,02\% $v / v$, in terms of low molecular compounds released, was higher from that of rennet and lower from that of Fromase at the concentration of those enzymes used in cheesemaking. The intensity of protein degradation by the preparation IPM was dependent on the kind of cheese and was higher for edam and kortowski cheese as compared to tilsit cheese.

Key words

Cheese slurry - Proteolytic activity - Ripening acceleration - Rennet - Fromase Microbial preparation.

\section{INTRODUCTION}

Le but de notre étude était de déterminer, dans des conditions modèles, l'activité protéolytique des préparations destinées à accélérer l'affinage des fromages. Cette préparation produite par l'Institut de l'Industrie Laitière de Varsovie est un mélange d'enzymes des bactéries Streptococcus lactis, Lactobacillus casei et Penicillium, candidum (Kalinowski et Coll., 1975). Dans un précédent article (Zelazowska et Coll., 1984) nous avons rapporté les résultats de nos études sur la dégradation de la caséine isoélectrique et de ses fractions par la préparation. Les résultats de ces études ont démontré que la préparation utilisée en quantité de $0,02 \% \mathrm{v} / \mathrm{v}$ dégrade la caséine en composés à poids moléculaire bas, solubles à $12 \%$ TCA d'une manière plus intensive que la présure. Il est en effet important du point de vue pratique que l'étude des préparations protéolytiques soit faite dans les conditions de leur utilisation habituelle.

Dans ce rapport, nous présentons nos études sur le processus d'hydrolyse des protéines dans la pâte des fromages par la préparation, appelée dans ce texte préparation IPM (de l'abrévation de la dénomination polonaise de l'Institut de l'Industrie Laitière de Varsovie).

\section{MATERIEL ET METHODES}

Le processus d'hydrolyse des protéines de fromage par la préparation IPM était comparé à celui obtenu par la présure (production polonaise : activité coagulante : 1/20 000) et par la préparation "Fromase " (Société Rapidase, France, activité coagulante : 1/100 000).

Les protéines des fromages Tilsit, Edam et Kortowski (fromage polonais à pâte demi-dure), servaient de substrats dans ces études. 
Les protéines de fromages étaient préparées pour les essais de la manière suivante : $100 \mathrm{~g}$ de fromage moulu étaient additionnés de $60 \mathrm{ml}$ d'une solution de la préparation enzymatique en question, et le mélange était homogénéisé. Les échantillons témoins étaient additionnés de $60 \mathrm{ml}$ d'eau distillée.

Les solutions de préparations enzymatiques ont été préparées de telle manière que la teneur en préparation IPM ajoutée à la pâte de fromage représente $0,02 \% \mathrm{v} / \mathrm{v}$, teneur optimale recommandée par le fabricant. Pour les préparations coagulantes les quantitées ajoutées au lait étaient celles habituellement employées pour la fabrication de fromage.

Après homogénéisation, les échantillons étaient conservés en petits sacs polyéthylènes à la température de $15^{\circ} \mathrm{C} \pm 2^{\circ} \mathrm{C}$. Les analyses étaient effectuées immédiatement après la préparation, puis après 4,7 et 10 jours de conservation.

Les teneurs en azote non-protéique étaient déterminées selon la méthode proposée par Schober (1961), les teneurs en composés solubles à $\mathrm{pH}$ 4,6 comme indiqué par Sode-Mogensen (1948) et les teneurs en composés peptidiques, d'après la méthode proposée par Boulanger et Biserte (1949).

Nous présentons dans cet article les résultats d'analyse des échantillons obtenus aussitôt après leur préparation et après 10 jours de conservation.

Après 10 jours de conservation, une analyse chromatographique des protéines était effectuée sur gel Sephadex G-100 Fine. Selon la méthode proposée par Tokito et Hosono (1968), on appliquait $200 \mathrm{mg}$ d'azote sur une colonne chromatographique K26/70 remplie de gel jusqu'à un volume de $350 \mathrm{ml}$. Les protéines étaient éluées avec la solution d'acétate de sodium à $1,3 \mathrm{M}, \mathrm{pH} 7,0$. On mesurait l'absorption de la lumière dans $20 \mathrm{ml}$ d'éluat, à la longueur d'onde de $200 \mathrm{~nm}$ (Zeiss VSU-1 - cuvette de 0,5 cm). Les éluats correspondant aux fractions successives étaient décantés et leur teneur en protéines était déterminée par la méthode de Kjeldahl. Le poids moléculaire des protéines était obtenu par calcul (Determann, 1968).

\section{RESULTATS}

Dans les échantillons témoins, les modifications protéolytiques se caractérisaient par l'accumulation de composés solubles à pH 4,6 dans le cas des protéines des fromages Kortowski et Edam, avec surtout une accumulation de composés non protéiques dans le cas des protéines des fromages Tilsit et Kortowski, et de composés peptidiques dans le cas des fromages Tilsit et Edam.

Dans les échantillons obtenus avec les préparations IPM et Fromase, on constatait une accumulation de composés peptidiques. Pour 
la Fromase, on observait en même temps l'accumulation de composés protéiques dans les échantillons du fromage Kortowski et celle de composés solubles dans un échantillon du fromage Tilsit.

Les résultats de protéolyse par la présure étaient semblables à ceux obtenus dans les échantillons témoins.

C'est la préparation Fromase qui présentait l'activité protéolytique la plus élevée. L'activité de la préparation IPM était comparable à celle de la présure.

Dans le cas du fromage Edam, c'était la préparation IPM et la présure qui libéraient le maximum de composés solubles, alors que dans le cas du fromage Tilsit, c'était la Fromase. Après 10 jours de conservation, le maximum d'écart de teneur en composés solubles avec l'échantillon témoin était respectivement de $3,4,3,7$ et 13,5 pour la préparation IPM, la présure et la Fromase (tab. 1). Les teneurs en composés solubles obtenus dans les échantillons de fromages Tilsit et Kortowski indiquent pour la préparation IPM une activité environ 3 fois plus faible que celle de la présure.

Pour les composés non-protéiques, les teneurs maximales libérées par la préparation IPM et la présure le sont dans le fromage Tilsit, alors que pour la Fromase, elles le sont dans le fromage Kortowski. Après 10 jours de conservation la différence maximale avec l'échantillon témoin est de 2,0 pour la préparation IPM, 1,4 pour la présure et 5,3 pour la Fromase.

Pour les composés peptidiques, toutes les préparations étudiées en libèrent le maximum dans le fromage Kortowski. L'activité de la préparation IPM est environ 3 fois plus élevée que celle de la présure alors qu'elle ne diffère que bien peu de celle de la Fromase. La libération de composés peptidiques dans le fromage Tilsit et dans l'Edam par la préparation IPM et la présure est insignifiant (tab. 1).

L'analyse chromatographique sur gel Sephadex G-100 Fine démontre également que dans les échantillons additionnés de préparations enzymatiques, le processus d'hydrolyse des protéines s'effectue d'une manière tout à fait différente de celle des échantillons témoins.

Dans ces échantillons témoins, les protéines des fromages Tilsit et Edam étaient réparties en 4 fractions, et celles du fromage Kortowski en 3 fractions (fig. 1). La préparation IPM était plus active vis-à-vis des protéines du fromage Edam puisqu'on obtenait 5 fractions contre 3 fractions pour les fromages Tilsit et Kortowski. La présure s'est montrée la plus active vis-à-vis des protéines du fromage Kortowski avec 4 fractions obtenues. La Fromase était plus active sur les protéines de Tilsit où on obtenait 6 fractions.

En considérant le poids moléculaire, la caractéristique des fractions a indiqué que, dans le cas de la préparation IPM, la quantité la plus élevée de composés à poids moléculaire élevé était présente dans l'échantillon de fromage Tilsit et la quantité la plus basse dans le fromage Kortowski (tab. 2). 


\section{TABLEAU 1}

Teneurs des fromages : Tilsit, Edam et Kortowski en produits de dégradation des protéines, immédiatement après la fabrication et après 10 jours d'incubation avec les préparations protéolytiques étudiées

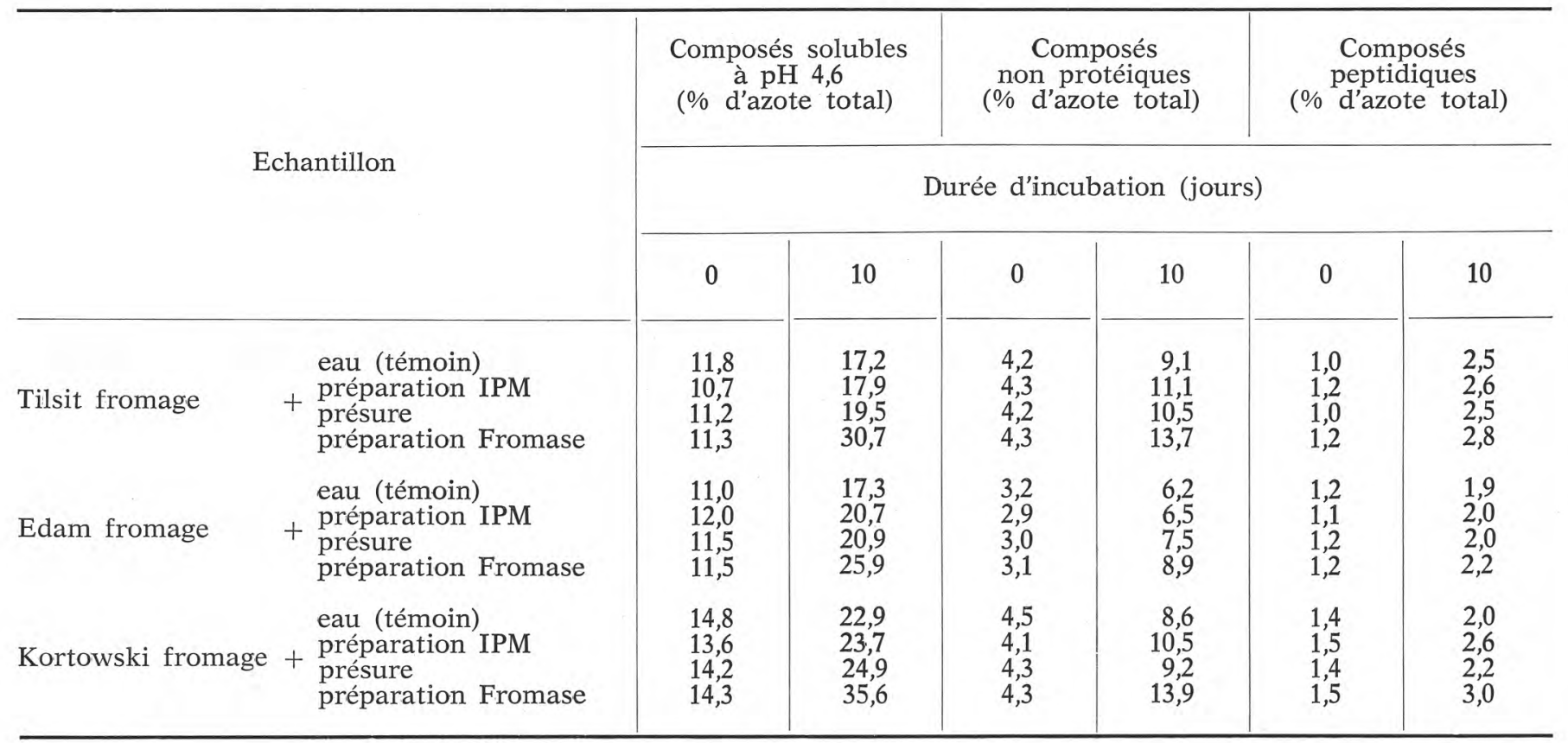


THLSIT
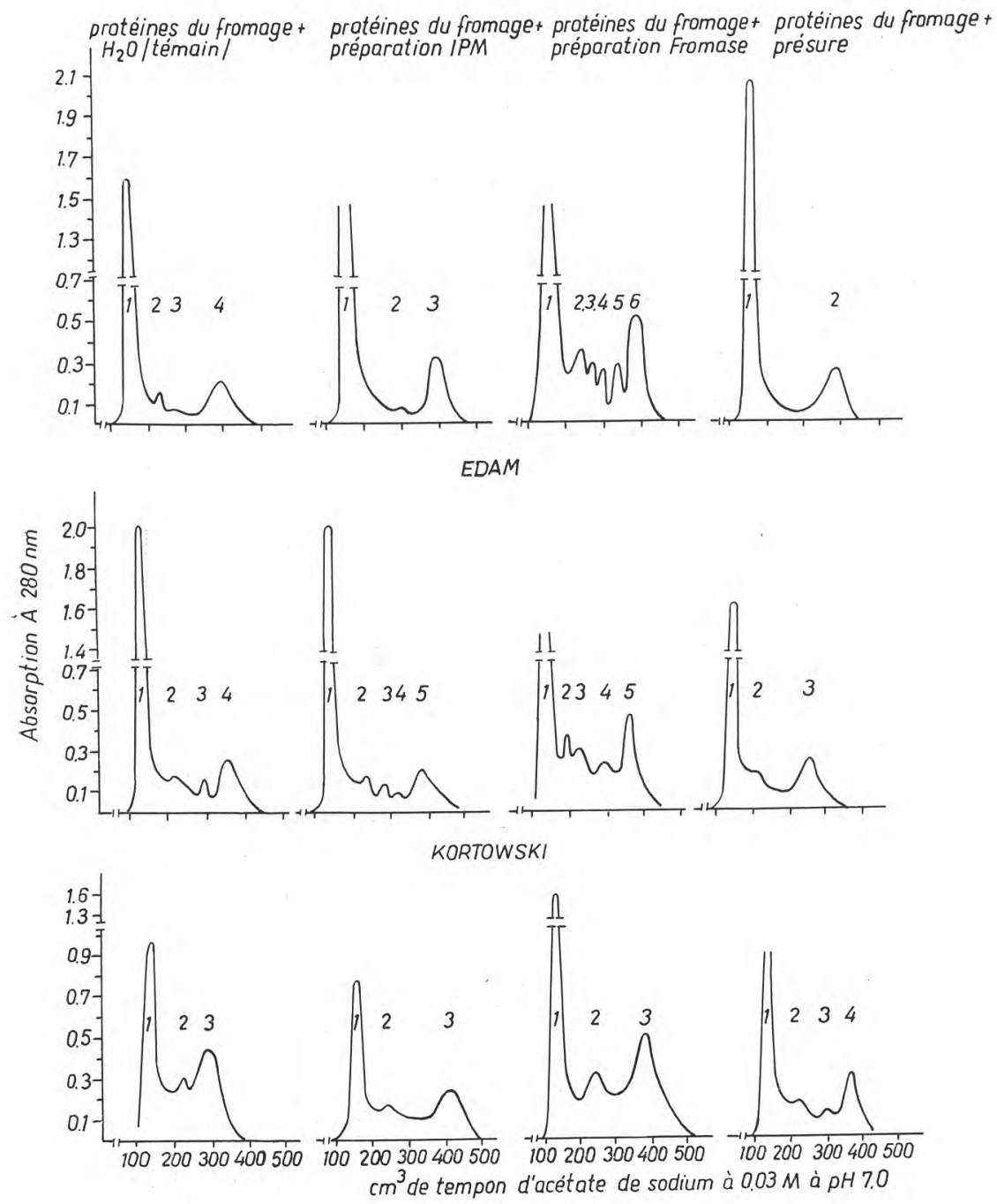

fig. 1

Répartition chromatographique sur le gel Sephadex G-100 des protéines des fromages étudiés.

Les composés à poids moléculaire moyen, dont la teneur était la plus élevée dans l'échantillon de fromage Kortowski, étaient absents dans le fromage Tilsit. Le taux de composés à bas poids moléculaire était le plus élevé pour les protéines du fromage Kortowski. 
TABLEAU 2

Répartitions chromatographiques sur le gel Sephadex G-100 des protéines des fromages Tilsit, Edam et Kortowski après 10 jours d'incubation avec les préparations protéolytiques étudiées

\begin{tabular}{|c|c|c|c|c|}
\hline \multirow{4}{*}{\multicolumn{2}{|c|}{ Echantillon }} & \multicolumn{3}{|c|}{ Pourcentage des fractions } \\
\hline & & à poids moléculaire & $\begin{array}{l}\text { à poids moléculaire } \\
\text { moyen }\end{array}$ & $\begin{array}{c}\text { à poids moléculaire } \\
\text { bas }\end{array}$ \\
\hline & & \multicolumn{3}{|c|}{ A poids moléculaire en mille de daltons } \\
\hline & & $100-35$ & $35-10$ & 10 \\
\hline Tilsit fromage & $\begin{array}{l}\text { eau (témoin) } \\
+ \text { préparation IPM } \\
\text { présure } \\
\text { préparation Fromase }\end{array}$ & $\begin{array}{l}59,64 \\
61,13 \\
69,14 \\
20,23\end{array}$ & $\frac{21,54}{36,98}$ & $\begin{array}{l}17,82 \\
38,66 \\
30,48 \\
42,79\end{array}$ \\
\hline Edam fromage & $\begin{array}{l}\text { eau (témoin) } \\
+\begin{array}{l}\text { préparation IPM } \\
\text { présure } \\
\text { préparation Fromase }\end{array}\end{array}$ & $\begin{array}{l}40,54 \\
44,32 \\
38,26 \\
24,54\end{array}$ & $\begin{array}{l}33,93 \\
23,34 \\
32,56 \\
47,13\end{array}$ & $\begin{array}{l}25,52 \\
32,14 \\
29,18 \\
28,13\end{array}$ \\
\hline Kortowski fromage & $\begin{array}{l}\text { eau (témoin) } \\
+ \text { préparation IPM } \\
\text { présure } \\
\text { préparation Fromase }\end{array}$ & $\begin{array}{l}59,79 \\
20,16 \\
24,95 \\
11,32\end{array}$ & $\begin{array}{r}8,49 \\
38,68 \\
53,15 \\
28,65\end{array}$ & $\begin{array}{l}31,70 \\
41,11 \\
21,90 \\
60,02\end{array}$ \\
\hline
\end{tabular}


Dans les échantillons additionnés de présure, la fraction des composés à poids moléculaire élevé était au même niveau que celle obtenue dans les échantillons additionnés de préparation IPM, tandis que pour les composés à poids moléculaire moyen et bas, les taux les plus élevés y étaient trouvés pour les protéines du fromage Kortowski.

Dans les échantillons additionnés de Fromase, le taux des composés à poids moléculaire élevé et moyen était le plus élevé pour les protéines du fromage Edam, tandis que celui des composés à bas poids moléculaire était maximum pour les protéines du fromage Kortowski.

\section{DISCUSSION ET CONCLUSION}

Le processus de dégradation des protéines dépendait à la fois de la préparation enzymatique utilisée et du fromage employé comme substrat. La tendance qu'ont les différentes préparations protéolytiques à dégrader les protéines plutôt en composés solubles ou en composés non protéiques ou en composés peptidiques témoigne de différences d'activité spécifique.

Sur la base de l'analyse chimique, on peut dire que, indépendamment de la variété de fromage, la préparation IPM a une activité protéolytique inférieure à celle de la Fromase et de la présure pour la production de composés solubles à $\mathrm{pH} 4,6$. En ce qui concerne la libération de composés non-protéiques, l'activité de la préparation IPM dépend de la variété de fromage et est en général plus élevée que celle de la présure, tout en approchant celle de la Fromase. Ces résultats confirment ceux obtenus par d'autres auteurs qui ont montré que l'hydrolyse des protéines de fromage par la présure produit des composés à poids moléculaire élevé (Green, 1977, Phelan, 1977), tandis que l'hydrolyse par la Fromase est plus intense et moins spécifique que celle de la présure (Reps et Coll., 1978). L'aptitude de la préparation IPM à hydrolyser les protéines en composés non-protéiques et peptidiques démontre que cette préparation contient des enzymes appartenant aux groupes des dipeptidases, carboxypeptidases et aminopeptidases.

Sur la base de l'analyse chromatographique, on peut constater que c'est dans le fromage Kortowski que la préparation IPM hydrolyse le maximum de protéines en composés de poids moléculaire moyen et bas.

La dégradation la plus intense des protéines par la présure en composés de poids moléculaire moyen, était observée dans l'échantillon du fromage Kortowski et dans l'échantillon de fromage Tilsit pour les composés de poids moléculaire bas. Pour la Fromase on obtient le maximum de composés de poids moléculaire moyen dans 
le fromage Edam et le maximum de composés de poids moléculaire bas dans le fromage Kortowski.

Les résultats de l'analyse chimique et chromatographique ont démontré que les propriétés protéolytiques de la préparation IPM dépendaient de la variété de fromage et différaient de celles des préparations coagulantes en ce qui concerne les produits de dégradation profonde des protéines obtenus. On peut donc en conclure que l'aptitude à accélérer l'affinage de la préparation IPM sera variable selon le fromage considéré. D'après nos résultats, on peut penser que l'accélération de l'affinage sera plus élevée pour les fromages Kortowski et Edam qu'elle ne le sera pour le fromage Tilsit. Pourtant, il faut indiquer qu'il peut être erroné de considérer l'étendue de la dégradation comme le critère unique de la maturité. D'après Kosikowski et Mocquot (1958), un équilibre défini entre les produits de dégradation des protéines et ceux de la matière grasse est la condition nécessaire à l'obtention de propriétés organoleptiques régulières. Une modification de cet équilibre peut être nuisible pour la qualité des fromages, ou bien peut faire unifier des valeurs sensorielles. Green (1977) et Phelan (1977) indiquent déjà que les succédanés de présure, qui se caractérisent par une valeur élevée du rapport entre l'activité protéolytique et l'activité de coagulation, ne peuvent pas être employés pour la fabrication des fromages.

Il est vrai que cette méthode d'étude de l'activité protéolytique que nous avons adoptée dans le présent travail, favorise les modifications enzymatiques, ce qui peut causer une altération des valeurs de qualité. Elle a cependant une valeur pratique incontestable pour définir l'utilité des préparations enzymatiques dans la fabrication des fromages (Kosikowski et Iwasaki, 1975, Kristoffersen et Coll., 1967).

\section{Bibliographie}

Boulanger (P.), Biserte (G.) (1949). - Chromatographie de portage des aminoacides libres du plasma sanguin. Bull. Soc. Chimie Biol., 31, 696.

Determann (H.) (1968). - Gel chromatography. Springer-Verlag, New York Inc.

GREEN (M.L.) (1977). - Review of the progress of dairy science : milk coagulants. J. Dairy Res., 44, 159.

Kalinowski (L.), Bijok (F.), Frackiewicz (E.), Pisarek (J.), Soltys (W.), Szadkowska (M.), TomaszewSKa (J.), Janiszewska (J.) (1975). - Otrzymywanie i zastosowanie preparatow enzymatycznych w serowarstwie. Inst. Przem. Mlecz (non publié).

Kosikowski (F.V.), Mocovot (G.) (1958), - Advances in cheese technology. F.A.O. Agric. Study No 38, Rome.

KosIKowSKI (F.V.), IWASAKI (T.) (1975). - Changes in Cheddar cheese by commercial enzyme preparation. J. Dairy Sci., 58, 963.

Kristoffersen (T.), Mikolajcik (E.M.), Gould (I.A.) (1967). - Cheddar cheese flavour. IV, Directed and accelerated ripening process. J. Dairy Sci., 50, 292. 
Phelan (J.A.) (1977). - Milk coagulants, a critical review. Dairy Ind. International, 42, 50.

Reps (A.), Poznanski (S.), Jedrychowski (L.), Zelazowska (H.), Babuchowski (A.) (1978). - Characterization of the microbial rennet Fromase. Milchwiss., $33,34$.

Sephadex gel filtration in theory and practice. Pharmacia Fine Chemicals, Sweden, 1970.

SCHOBER (R.K.) (1961). - Anwendung der "Finger-Abdruck Methode 》 auf die Kenzeichnung von Käsesorten durch ihre proteolytischen Inhaltsstoffe. Milchwiss., 16, 142.

Sode-Mogensen (T.) (1948). - Determination of the degree of proteolytic decomposition in cheese with special reference to the formol titration. Meddelande No 21, Fran Statens Mejeriförsök, Alnarp-Akarp.

Tокіто (F.), Hosono (A.) (1968). - An investigation about limburger cheese ripening by gel filtration on Sephadex. Milchwiss., 23, 758.

Zelazowska (H.), Reps (A.), JedRychowski (L.), JaRmUl (I.). - Degradacja wszystkich bialek mleka, kazeiny izoelektrycznej i jej frackcji przez preparat mikrobiologiczny do przyspieszania dojrzewania serow. Rocz. Inst. Przem. Mlecz., 1984 (sous presse). 